\title{
Bevacizumab Versus Anti-preeclamptic Drugs: Evaluation With Three-dimensionally Co-cultured Human Mini Tumors
}

\author{
CHEN PAN ${ }^{1,2}$, KENJI ONDA $^{1}$ and TOSHIHIKO HIRANO ${ }^{1}$ \\ ${ }^{1}$ Department of Clinical Pharmacology, School of Pharmacy, \\ Tokyo University of Pharmacy and Life Sciences, Tokyo, Japan; \\ ${ }^{2}$ Key Laboratory of Beijing for Identification and Safety Evaluation of Chinese Medicine, \\ Institute of Chinese Materia Medica, China Academy of Chinese Medical Sciences, Beijing, P.R. China
}

\begin{abstract}
Background/Aim: Both bevacizumab (BEV) and soluble fms-like tyrosine kinase-1 (sFlt-1) have demonstrated anti-angiogenic effects, thereby causing hypertension and proteinuria. We hypothesized that anti-preeclamptic drugs that combat the action of sFlt-1 may reduce BEV's antitumor efficacy. Materials and Methods: $3 D$ co-cultured human mini-tumors consisting of endothelial cells, fibroblasts, and cancer cells were developed. The influence of anti-preeclamptic drugs and BEV on the invasion of minitumors embedded in collagen gel was evaluated. Results: Mini-tumor spheroids that contained MDA-MB-231 cells showed higher invasion ability than spheroids with A549. Among the six anti-preeclamptic drugs investigated, only nicorandil enhanced the invasion of mini-tumors and inhibited the action of BEV. Glibenclamide, an ATP-sensitive potassium channel inhibitor, completely quenched the action of nicorandil on mini-tumors. Conclusion: In the human mini-tumor model, nicorandil aggravated the invasion of mini-tumors. These data raise the possibility that concomitant use of nicorandil counteracts the efficacy of BEV therapy.
\end{abstract}

Tumor angiogenesis is essential for the growth, invasion, and metastatic spread of malignant tissues. Angiogenesis inhibitors have emerged as an effective therapy for reducing proliferation of solid tumors by inhibiting endothelial angiogenesis. Bevacizumab (BEV), a humanized monoclonal antibody, is an angiogenesis inhibitor that inhibits vascular endothelial growth factor (VEGF) activity, and thereby

Correspondence to: Kenji Onda, Department of Clinical Pharmacology, School of Pharmacy, Tokyo University of Pharmacy and Life Sciences, 1432-1 Horinouchi, Hachioji, Tokyo 192-0392, Japan. Tel: +81 426765795, e-mail: knjond@ toyaku.ac.jp

Key Words: Bevacizumab, preeclampsia intervention, nicorandil, ATP-sensitive potassium channel, mini-tumor, invasion. exhibits clinical benefit in the treatment of several common cancers including colorectal, breast, ovarian, or non-small cell lung cancer (1). However, side-effects of BEV therapy such as hypertension and proteinuria are often observed and could limit the continuation of therapy when the grade is severe (2).

In the field of obstetrics, preeclampsia (PE), which is defined by the onset of both hypertension and proteinuria after 20 weeks of gestation, is one of the leading causes of pregnancy-related maternal deaths worldwide (3). Although the cause of PE is incompletely understood, recent studies have demonstrated the role of soluble fms-like tyrosine kinase (sFlt-1), the placental-derived anti-angiogenic factor in PE pathophysiology (4). Studies have shown that: i) sFlt1 is excessively released into the circulating maternal serum, and plays an anti-angiogenic role by trapping it's ligands such as VEGF and placental growth factor (PlGF) (4). ii) Administration of viral transfer of sFlt-1 is enough to cause hypertension and proteinuria (4). iii) Adsorption of sFlt-1 protein in serum by apheresis in patients with severe PE ameliorates disease symptoms (5). Thus, these studies have clearly shown that sFlt-1 acts as a pathogenic molecule, inducing PE symptoms exemplified by hypertension and proteinuria (6).

To date, delivery of fetus and placenta is still the only definitive cure for PE. Besides conventional anti-hypertensive therapy, there have been some new candidate drugs that are repurposed based on the recent recognition of sFlt-1 and proposed as novel strategy for prevention or treatment of PE. In a transgenic pregnant $\mathrm{PE}$ mouse model where human sFlt-1 was specifically overexpressed in placenta, pravastatin induced PIGF and ameliorated PE symptoms (7). Esomeprazole has also been shown to inhibit the rise in blood pressure in the same PE mouse model and reduce sFlt- 1 and soluble endoglin (sENG) production in vitro (8). Sildenafil is reported to enhance nitric oxide (NO) signaling and reverse the sFlt-1-induced hypertension in an adenovirus mediated PE mouse model (9). Nicotinamide is known to inhibit increased blood pressure in 
sFlt-1-overexpressing pregnant mice (10). Metformin, which was recently shown to have a preventative effect on PE in human (11), decreased both sFlt-1 and sENG secretion and had angiogenic effects (12). Nicorandil was shown to have a significant positive therapeutic efficacy on the disorders in NG-Nitro-L-arginine methyl ester (L-NAME) treated PE mice (13).

Both BEV and sFlt-1 are anti-angiogenic factors and have similar anti-VEGF actions, thereby causing hypertension and proteinuria. Besides, it is recognized that hypertension and proteinuria caused by BEV treatment are called as "preeclampsia-like syndrome" in cancer patients (14). Thus, these understandings have led us to hypothesize that the clinically used medicines that have been shown to antagonize the PE phenotype induced by sFlt-1 in vivo would attenuate hypertension and proteinuria, and furthermore, the anti-tumor efficacy of BEV.

In order to verify our hypothesis, we directly evaluated the effect of anti-PE drugs in combination with BEV on human tumors. We adopted a three-dimensional (3D) human minitumor system which consists of endothelial cells, fibroblasts, and cancer cells to mimic the microenvironment of tumors in vivo (15). The ability of mini-tumor spheroids to expand or invade in collagen gel was assessed, and the pharmacological effect of anti-PE drugs in combination with $\mathrm{BEV}$ on the ability of tumor spheroid expansion in vitro was examined.

\section{Materials and Methods}

Drugs and reagents. Bevacizumab (BEV, anti-VEGF, humanized Antibody) was purchased from Biovision incorporated (Milpitas Boulevard, CA, USA). Human recombinant vascular endothelial growth factor $165\left(\mathrm{VEGF}_{165}\right)$, pravastatin sodium, sildenafil, nicotinamide, nicorandil, diazoxide, and glibenclamide were obtained from Fujifilm Wako Pure Chemical Corporation. Esomeprazole sodium was purchased from Abcam. Dimethyl sulfoxide (DMSO) was used to dissolve esomeprazole, sildenafil, nicorandil, diazoxide, and glibenclamide. The final concentration of DMSO was kept at $0.1 \%$ throughout the study. Pravastatin, nicotinamide, and metformin were dissolved in medium.

Cell culture. Primary human umbilical vein endothelial cells (HUVECs, PromoCell, the passage number $<6$ ) were cultured in Medium 199 (Thermo Fisher Scientific, Tokyo, Japan) containing $20 \%$ fetal bovine serum (FBS, Sigma-Aldrich, Lot BCBT3482, Tokyo, Japan), endothelial cell growth supplement (ECGS, SigmaAldrich) $(0.02 \mathrm{mg} / \mathrm{ml})$, heparin (Sigma-Aldrich) $(0.1 \mathrm{mg} / \mathrm{ml})$, and $1 \%$ Antibiotic-Antimycotic (Anti-anti, Thermo Fisher Scientific). MDA-MB-231 breast cancer cells (American Type Culture Collection) are cultured in MEM- $\alpha$ (Thermo Fisher Scientific) with $10 \%$ FBS and 1\% Anti-anti, and normal human dermal fibroblasts (PromoCell) and A549 lung cancer cells (American Type Culture Collection) were cultured in DMEM (Thermo Fisher Scientific). Cells were maintained at $37^{\circ} \mathrm{C}$ in a humidified $5 \% \mathrm{CO}_{2}$-containing atmosphere.
Co-culture of three different human cells to create mini-tumor by hanging drop technique. The Hanging Drop System (InSphero AG, Switzerland) was used to create 3D spheroid mini-tumors. A total of $40 \mu \mathrm{l}$ of HUVECs culture medium which contains 10,000 HUVECs, 20,000 fibroblasts and 10,000 cancer cells (A549 or MDA-MB-231) was added to each well of a GravityPLUS ${ }^{\text {TM }}$ plate to create the $3 \mathrm{D}$ spheroid mini-tumors and cultured for 4 days.

Invasion assay with $3 D$ co-cultured human mini-tumors. Two different types of human mini-tumors were made. HFA which consists of HUVECs, fibroblasts and A549, and HFM which consists of HUVECs, fibroblasts and MDA-MB-231 cells, respectively. After 4 days of co-culture, both HFA and HFM spheroids were transferred to each well of a 96-well plate that contained drugs to be tested in collagen gel and medium. Collagen gel was prepared by Collagen gel matrix (Cellmatrix, Nitta Gelatin Inc., Osaka, Japan), according to manufacturer's instruction. After setting collagen gel on the base layer, mini-tumor spheroids were transferred onto the base layer in each well of the receiving plate. After removal of excess medium, the second layer of the gel was prepared. Finally, the HUVECs culture medium was added to each well. Mini-tumor spheroids were cultured at $37^{\circ} \mathrm{C}$ in a $5 \% \mathrm{CO}_{2}$ humidified atmosphere.

Images of 3D mini-tumor spheroids were captured using Operetta CLS High-Content Analysis system (PerkinElmer Inc., Waltham, MA, USA). Images of different spots in a well were taken at $20 \times$ magnitude to increase resolution and were reconstituted by an operational software (Harmony 4.6, PerkinElmer) to generate an appropriate picture to cover the whole spheroid. The area of growth and invasion on days 0 to 4 was quantified by ImageJ software (16).

Fluorescent staining of HUVECs, fibroblasts and MDA-MB-231 cells. To identify which cell type in the co-cultured human minitumor was affected by drug treatment, 5- or 6-( $\mathrm{N}$-succinimidyloxycarbonyl)-fluorescein 3',6'-diacetate (CFSE) (DOJINDO, Kumamoto, Japan) staining was performed. HUVECs, fibroblasts or MDA-MB-231 cells were independently stained with CFSE $(5 \mu \mathrm{M})$ for $30 \mathrm{~min}$ at $37^{\circ} \mathrm{C}$, and then cells were washed two times with HUVECs cultured medium. One kind of labeled cells was mixed with the other two types of unstained cells and applied for co-culture under the hanging drop system as described above. Spheroids created were embedded in collagen gel and cultured. Fluorescent images were obtained by Operetta CLS at $496 \mathrm{~nm} / 516 \mathrm{~nm}$.

Statistical analyses. All data were expressed as mean \pm SEM. In the invasion assay, the number of samples in each group represented the number of spheroids $(n=3-6)$. For comparisons among 3 or more groups, One-way ANOVA followed by Dunnett or Tukey, where appropriate, was used for evaluation of data. Statistical analysis was performed using GraphPad Prism 7.0 (CA, USA).

\section{Results}

Establishment of human mini-tumor spheroids and validation of invasion ability under angiogenic and anti-angiogenic stimuli. Before we evaluated the influence of anti-PE drugs, we assessed the growth of the mini-tumor spheroids in collagen gel in response to angiogenic and anti-angiogenic stimuli, and then compared the growth ability between the 
A
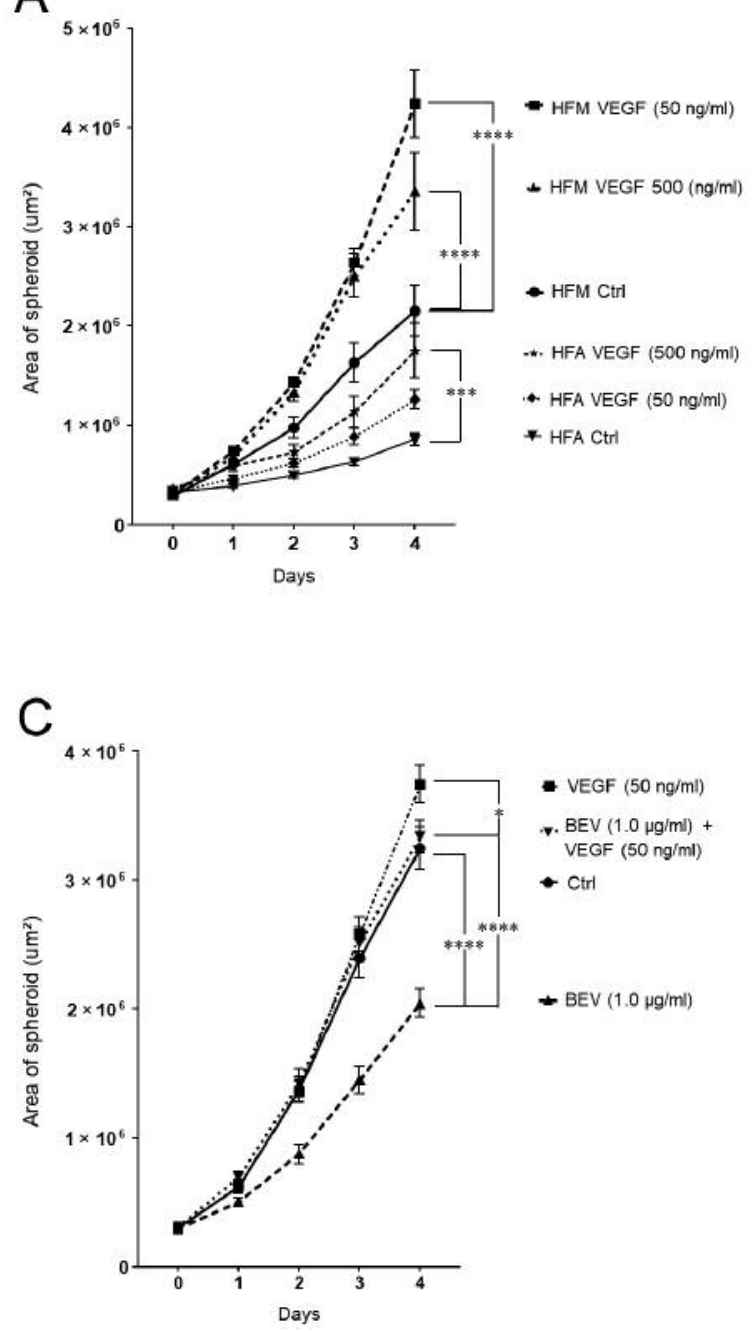

B

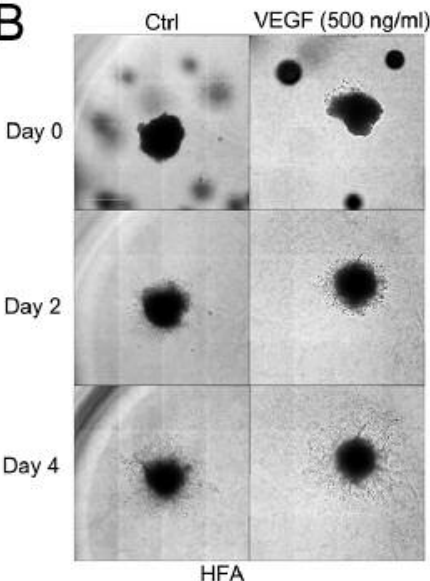

(HUVECs, fibroblasts, A549 cells )

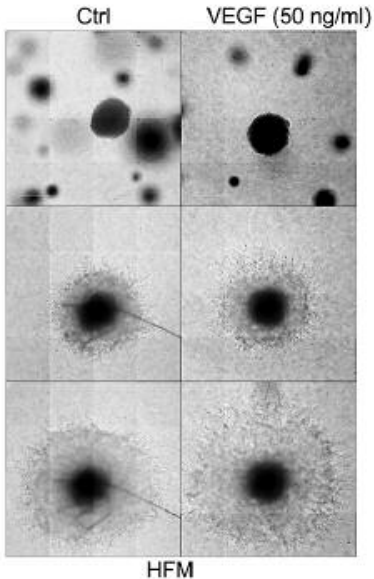

(HUVECs, fibroblasts, MDA-MB-231 cells )

D

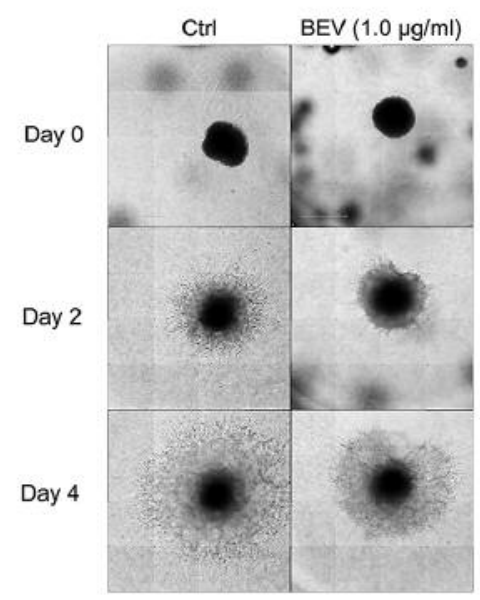

Figure 1. Comparison of the invasive ability of two human mini-tumor spheroids embedded in collagen gel and the influence of recombinant VEGF 165 and bevacizumab. A) Time-dependent change in the invasion areas of human mini-tumor spheroids (HFA: HUVECs, fibroblasts, and A549 cells or HFM: HUVECs, fibroblasts, and MDA-MB-231) that are treated with recombinant vascular endothelial growth factor (VEGF 165$)(50,500 \mathrm{ng} / \mathrm{ml})$ in collagen gel. B) Bright field images of HFA (left) and HFM (right) incubated in collagen gel with VEGF $165(50,500 \mathrm{ng} / \mathrm{ml})$ on days 0 , 2 , and 4. C) Time-dependent change in the areas of HFM spheroid treated with VEGF $165(50 \mathrm{ng} / \mathrm{ml})$ and/or bevacizumab $(B E V)(1.0 \mu \mathrm{g} / \mathrm{ml})$. D) Images of HFM spheroids in collagen gel treated with BEV $(1.0 \mu \mathrm{g} / \mathrm{ml})$ observed on days 0,2 , and 4 . Significant reduction of spheroid invasion was observed by BEV treatment on day 4. Mean \pm SEM, $n=6 . *, * *, * * *$ and $* * * *$ indicate $p<0.05,0.01,0.001$, and 0.0001 , respectively.

two types of mini-tumor spheroids, i.e., HFA (HUVECs, fibroblasts, and A549 cells) and HFM (HUVECs, fibroblasts, and MDA-MB-231 cells) (Figure 1A and B).

The invasion images of the $3 \mathrm{D}$ mini-tumor spheroids in collagen gel were monitored from day 0 to day 4 . The area of invasion of both mini-tumors (HFA and HFM) increased over time. On day 4, the size of HFM grew 7.2-fold as compared to the original size on day 0 , whereas the size of HFA grew 2.6-fold as compared to day 0 . The size of HFM on day 4 was 2.5-fold larger than that of HFA at the same time point (Figure 1A). The invasion area of HFM significantly increased by
$\operatorname{VEGF}_{165}$ stimulation (50 and $500 \mathrm{ng} / \mathrm{ml}$ ) as compared with the control on day $4(p<0.0001)$ (Figure $1 \mathrm{~A})$, whereas there was a little increase in the invasion of HFA mini-tumor under a high concentration of $\operatorname{VEGF}_{165}(500 \mathrm{ng} / \mathrm{ml})(p<0.001)$, suggesting that HFM spheroids are more invasive and responsive to VEGF stimulation than HFA (Figure 1A and B).

Next, by using the HFM spheroids, the effect of BEV on the invasion of HFM mini-tumor spheroids cultured for 4 days (Figure 1C and D) was evaluated. The invasion area of HFM spheroids on day 4 significantly decreased by $37 \%$ upon treatment with BEV $(1.0 \mu \mathrm{g} / \mathrm{ml})(p<0.0001)$. Again, 
$\mathrm{VEGF}_{165}(50 \mathrm{ng} / \mathrm{ml})$ stimulation significantly increased the invasion area of HFM $(p<0.01)$, which was almost completely quenched by BEV treatment $(1.0 \mu \mathrm{g} / \mathrm{ml})$ $(p<0.05)$ (Figure 1C and D).

Evaluation of anti-preeclamptic drugs in combination with $B E V$ on the invasion of HFM spheroid. By use of the HFM mini-tumor spheroid, we evaluated the effects of six different clinical drugs that are proposed to be therapeutic for PE on the invasion of HFM spheroids (Figure 2A-G). Pravastatin, esomeprazole, sildenafil, nicotinamide, metformin, and nicorandil in combination with BEV were evaluated for their pharmacological efficacies on the invasion of the HFM minitumor model.

Treatment of HFM with pravastatin $(10,50 \mu \mathrm{M})$, sildenafil $(2 \mu \mathrm{M})$, nicotinamide $(0.1,1 \mathrm{mM})$, or metformin $(0.1,1 \mathrm{mM})$ for 4 days decreased the invasion area of HFM spheroids, and except for sildenafil the others caused a decrease in a concentration-dependent manner (Figure 2A, C, D and E). Esomeprazole tended to decrease the area of invasion, but the effect was not statistically significant (Figure 2B). When HFM was treated with anti-PE drugs in combination with BEV $(1.0 \mu \mathrm{g} / \mathrm{ml})$, the inhibitory effect of pravastatin, esomeprazole, sildenafil, nicotinamide, and metformin was potentiated by the concomitant treatment with BEV (Figure $2 \mathrm{~A}-\mathrm{E})$. The combination effect of sildenafil and BEV was the most obvious (Figure 2C) $(p<0.001)$.

In contrast to the above drugs, nicorandil tended to enhance the HFM mini-tumor invasion in a dose-dependent manner after 4 days of culture (Figure $2 \mathrm{~F}$ and $\mathrm{G}$ ). When nicorandil was combined with $\mathrm{BEV}$, the invaded area of HFM that was suppressed by BEV was significantly increased by treatment with nicorandil $(5,50 \mu \mathrm{M})$ by 1.4 and 1.8-fold as compared to the BEV treated group, respectively $(p<0.05$ and $p<0.0001)$.

The role of ATP-sensitive potassium channel activation in nicorandil-induced stimulation of mini-tumor invasion. Nicorandil is an ATP-sensitive potassium $\left(\mathrm{K}_{\mathrm{ATP}}\right)$ channel activator. As previous studies have suggested that nicorandil has angiogenic properties (17), we hypothesized that $\mathrm{K}_{\text {ATP }}$ channel activation is associated with the promotion of minitumor invasion observed in our study. To investigate this hypothesis, the influences of diazoxide, a direct $\mathrm{K}_{\text {ATP }}$ channel activator, and glibenclamide, a $\mathrm{K}_{\text {ATP }}$ inhibitor, were examined on the 3D HFM mini-tumor spheroids.

Treatment of HFM spheroids with diazoxide $(10,20 \mu \mathrm{M})$ showed a modest increase in the area of invasion when cultured for 4 days. Additionally, the effect of diazoxide significantly counteracted the suppressive action of BEV on the invasion of HFM (Figure $3 \mathrm{~A}$ and B) $(p<0.05)$.

Then, the effect of nicorandil in combination with glibenclamide on our mini-tumor system was examined. The increase in the area of invasion of HFM by the treatment with nicorandil $(100 \mu \mathrm{M})$ was completely quenched by the presence of glibenclamide $(10 \mu \mathrm{M})$, while glibenclamide itself did not change the invasion area of the mini-tumor spheroids (Figure 3C and D). When the HFM spheroids were treated with diazoxide $(20 \mu \mathrm{M})$ in the presence of glibenclamide $(10 \mu \mathrm{M})$, the increase in the area of invasion of HFM spheroid treated with diazoxide was completely diminished by glibenclamide (Figure 3E and F).

Identification of the cell type which was affected by $K_{A T P}$ channel activators in the co-cultured human mini-tumors. Next, we aimed to identify which type of cells in the HFM spheroids that consist of endothelial cells, fibroblasts, and cancer cells was responsible for the drug effect. Each type of cells was stained with a CFSE fluorescent dye, and then three different HFM spheroids, where one of the three types of cells was CFSE-stained, were developed by hanging drop and cultured in collagen gel for 4 days. The relative area of CFSE stained-MDA-MB-231 cells was larger than the area of the other two types of cells (HUVECs and fibroblasts) under spontaneous growth for 4 days. When HFM spheroids were treated with nicorandil $(100 \mu \mathrm{M})$ or diazoxide $(20 \mu \mathrm{M})$, the relative area of MDA-MB-231 dominantly increased during the 4 days of culture, whereas there was no increase in the area of HUVECs or fibroblasts upon treatment with either drug (Figure 4A and B).

\section{Discussion}

Tumor spheroids co-cultured with endothelial and stroma cells have been used to mimic cellular interactions in the tumor microenvironment and for the evaluation of anticancer drugs and angiogenesis $(15,18)$. In our present study, we prepared two kinds of human mini-tumor spheroids, HFA and HFM that consist of HUVECs, fibroblasts, and tumor cells (A549 for HFA and MDA-MB231 for HFM, respectively) and used them for the assessment of invasion ability. We confirmed that the invaded area of mini-tumors in collagen gel clearly changed in response to angiogenic $\left(\mathrm{VEGF}_{165}\right)$ and antiangiogenic (BEV) stimuli (Figure 1).

Using this spheroid system, we evaluated the influence of the clinical or subclinical concentrations of six anti-PE drugs (pravastatin, esomeprazole, sildenafil, nicotinamide, metformin, and nicorandil), in the presence or absence of $\mathrm{BEV}$, on the invasion of mini-tumor spheroids. We conducted this examination based on the hypothesis that anti-PE drugs, which combat the action of sFlt-1, may also diminish the anti-cancer efficacy of BEV. To the best of our knowledge, there has been no such ideas proposed or validated.

The results demonstrated that, contrary to what we expected, five out of six drugs (pravastatin, esomeprazole, 

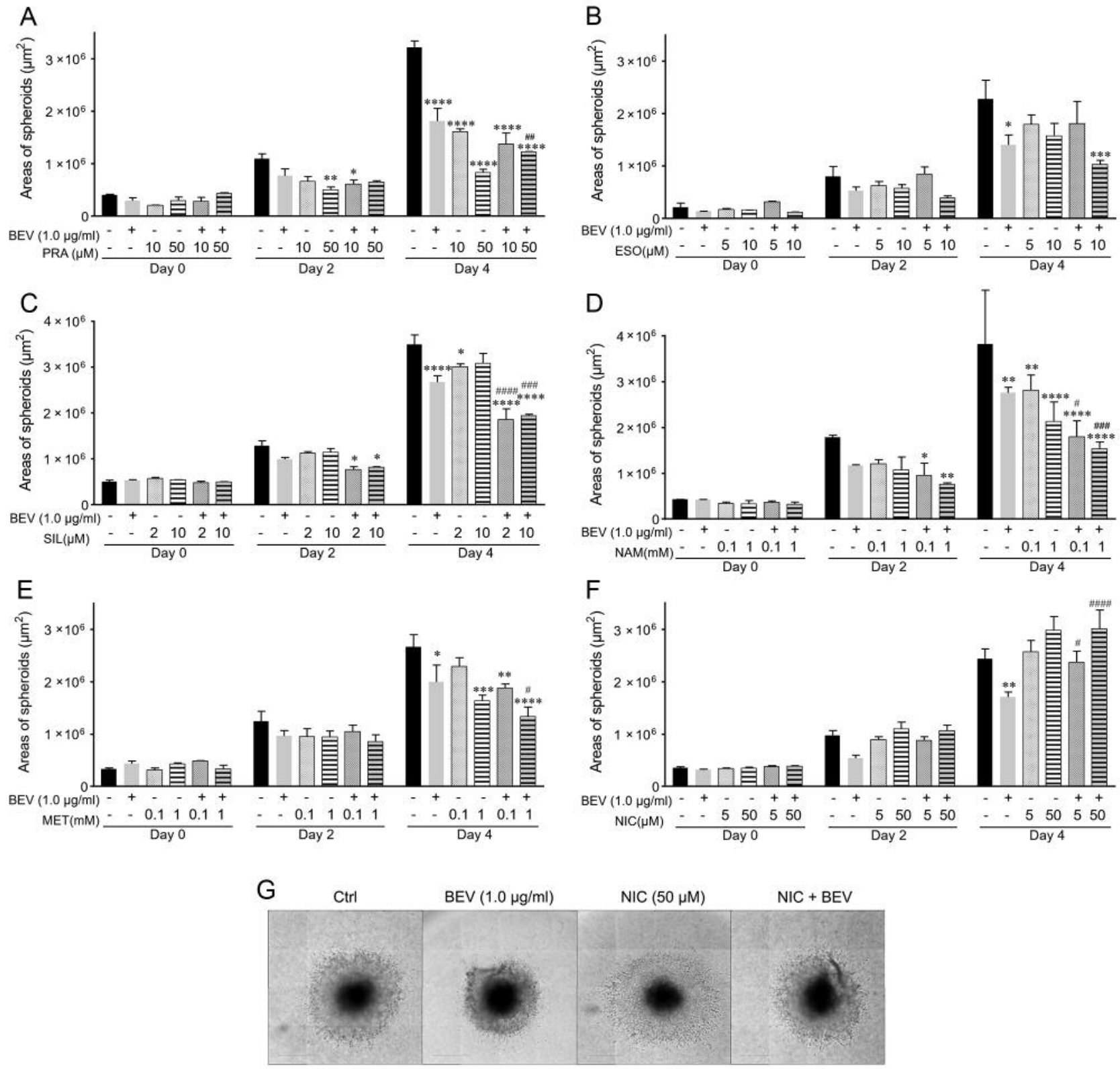

Figure 2. Evaluation of the effect of anti-preeclamptic drugs in combination with bevacizumab on the invasion area of HFM mini-tumor embedded in collagen gel. A) Pravastatin (PRA; 10, $50 \mu M, n=3)$, B) Esomeprazole (ESO; 5, $10 \mu M, n=3)$, C) Sildenafil (SIL; 2, $10 \mu M, n=3)$, D) Nicotinamide (NAM; 0.1, $1 \mathrm{mM}, n=3)$, E) Metformin (MET; 0.1, $1 \mathrm{mM}, n=3), F)$ Nicorandil (NIC; 5, $50 \mu M, n=6)$. Data represent mean \pm SEM. *, **, ***, and **** indicate $p<0.05,0.01,0.001$, and 0.0001, respectively as compared with the control on the same day. \#, \#\#, \#\#, and \#\#\#\# represent p<0.05, $0.01,0.001$, and 0.0001, respectively as compared with the BEV treated group on the same day. G) Bright field images of HFM spheroids treated with NIC $(50 \mu \mathrm{M})$ and/or BEV $(1.0 \mu \mathrm{g} / \mathrm{ml})$ on day 4.

sildenafil, nicotinamide, and metformin) showed suppressive effects on the invasion of the mini-tumor spheroids (Figure 2). The suppressive effects were more potent when they were used in combination with BEV, suggesting that these five drugs are able to potentiate the anti-tumor efficacy of BEV.
In relation to the previous studies on these five anti-PE drugs, there is evidence showing the angiogenic and anticancer properties of these five anti-PE drugs. No studies, as far as we understand, have been conducted using a 3dimensional human mini-tumor co-culture model. Pravastatin stimulates angiogenesis (7), whereas it inhibits tumor cell 


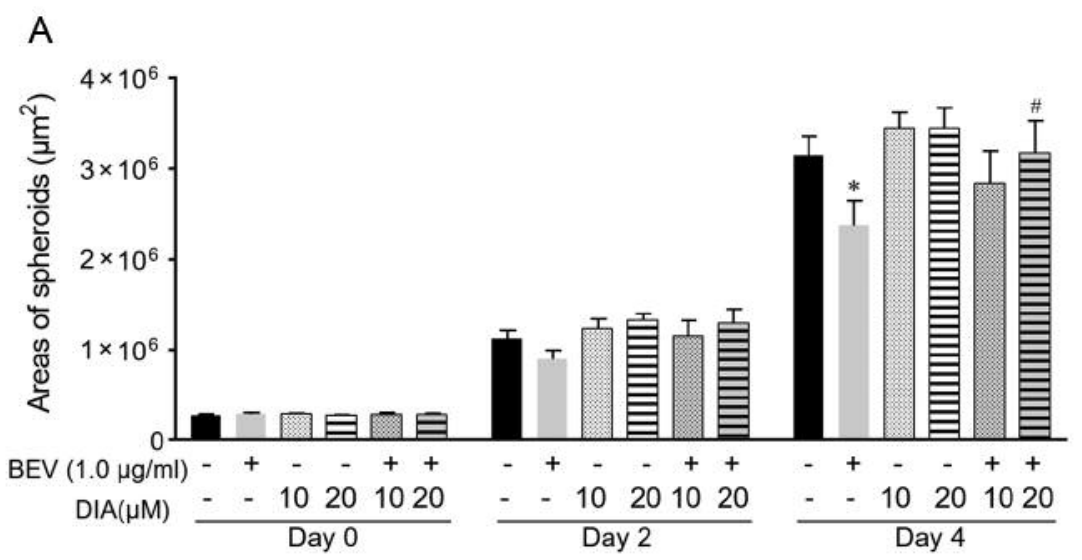

B
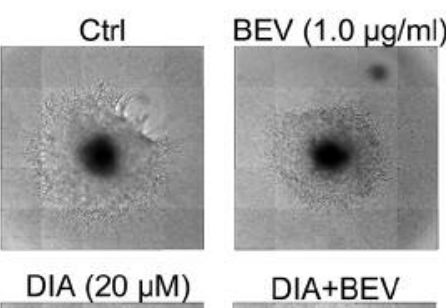

$\mathrm{DIA}+\mathrm{BEV}$
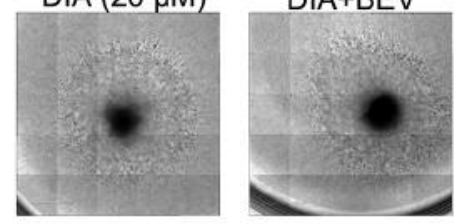

C

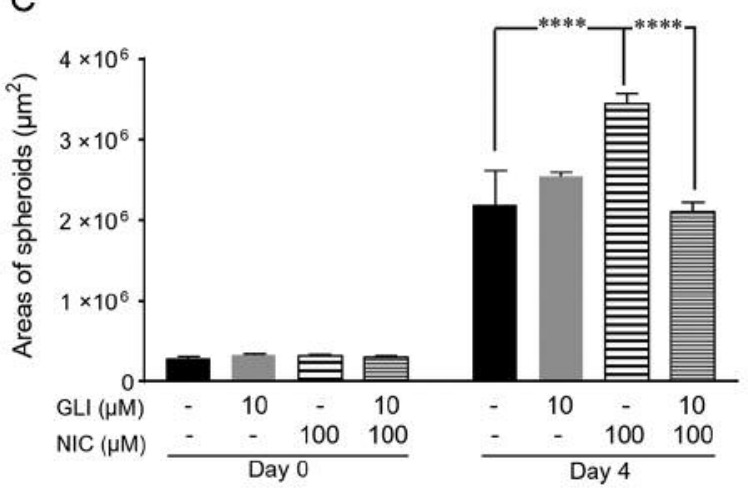

D
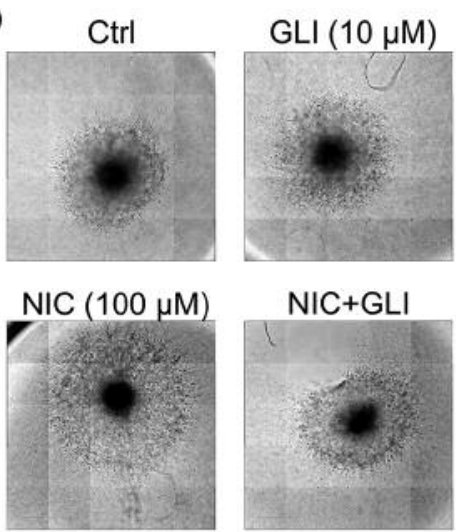

E

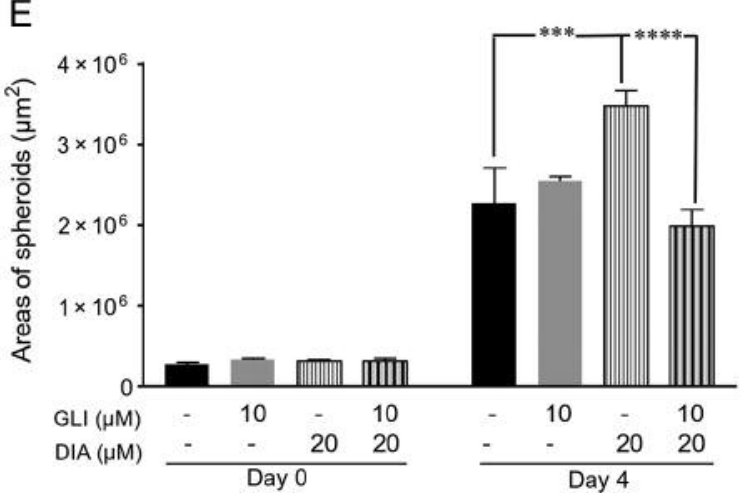

$\mathrm{F}$
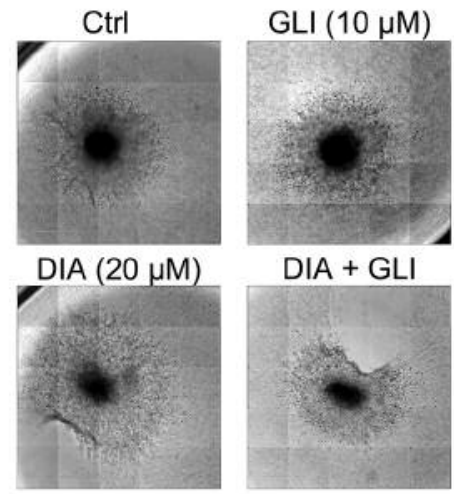

Figure 3. Involvement of ATP-sensitive potassium channel in the invasion of 3D HFM mini-tumor. A) Effect of diazoxide (DIA; 10, $20 \mu M)$, an ATPsensitive potassium channel activator on the invasion of HFM mini-tumor embedded in collagen gel $(n=6)$. B) Bright field images of HFM spheroids treated with DIA $(20 \mu \mathrm{M})$ and/or BEV $(1.0 \mu \mathrm{g} / \mathrm{ml})$ on day 4.C) Effect of nicorandil $(\mathrm{NIC} ; 100 \mu \mathrm{M})$ in the presence or absence of glibenclamide $(G L I ; 10 \mu M)$, an ATP-sensitive potassium channel inhibitor, on invasion of HFM mini-tumor embedded in collagen gel ( $n=6)$. D) Images of HFM spheroids treated with NIC $(100 \mu M)$ and/or GLI $(10 \mu M)$ captured on day 4. E) Effect of DIA $(20 \mu M)$ and GLI (10 $\mu M)$ on the invasion area of HFM spheroids $(n=6)$. F) Images of HFM spheroids treated with DIA $(20 \mu M)$ and/or GLI $(10 \mu M)$ captured on day 4.

proliferations and suppresses tumor growth in xenograft models $(19,20)$. Esomeprazole induces VEGF expression (8), while proton pump inhibitors have been reported to induce apoptosis in cancer cells and increase chemosensitivity of drug-resistance human solid tumors (21, 22). Sildenafil potentiates angiogenesis (9), while it enhances the sensitivity of certain types of cancer to chemotherapeutic drugs (23). It has been reported that nicotinamide induces apoptosis in 
A

\section{CFSE-stained cells}
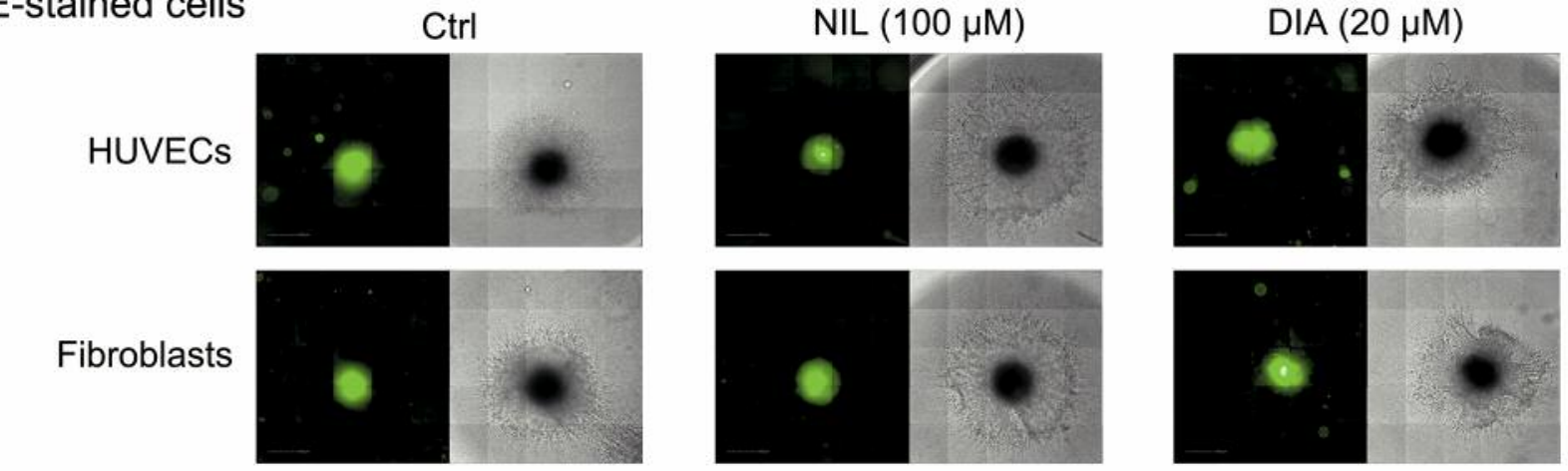

MDA-MB-231
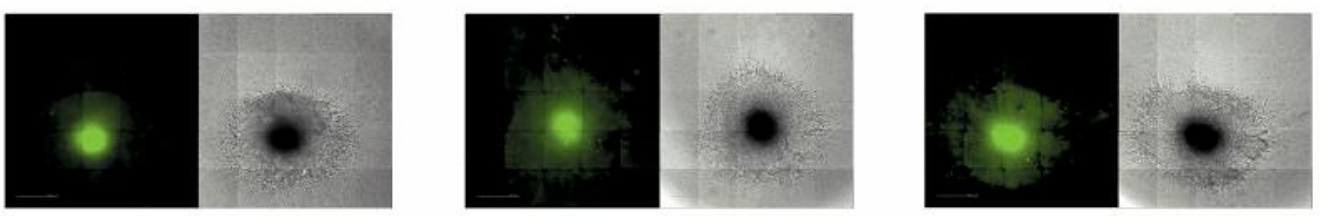

B

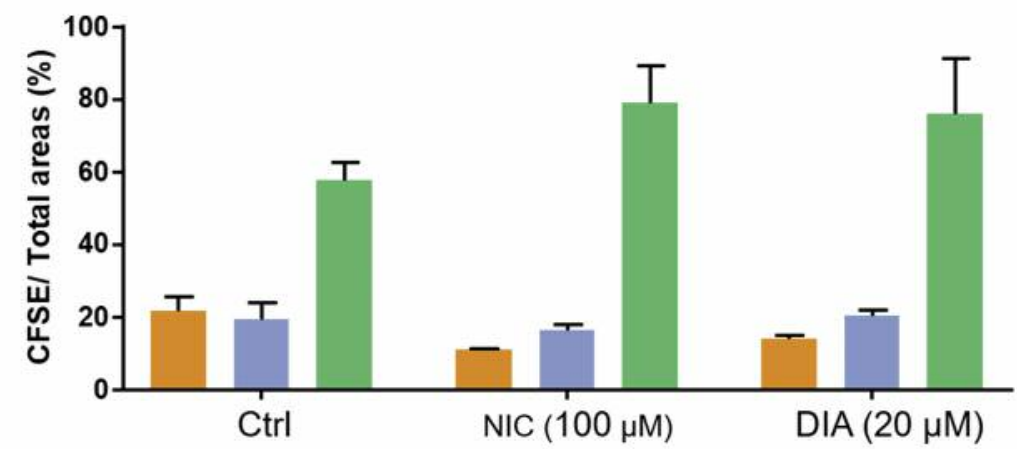

HUVECs

Fibroblasts

MDA-MB-231

Figure 4. Fluorescent staining of component cells and the influence of nicorandil and diazoxide treatment. A) HUVECs, fibroblasts, or MDA-MB231 cells were independently stained with CFSE, and three different mini-tumor spheroids were created where one of the cell types was stained. Both nicorandil (NIC; $100 \mu \mathrm{M})$ and diazoxide (DIA; $20 \mu \mathrm{M})$ enhanced HFM invasion under bright field observation, which was accompanied by the spread of MDA-MB-231 cells, but not of HUVECs or fibroblasts, on day 4. B) Quantification of the area of component cells stained with CFSE that are derived from the respective HFM spheroids on day $4(n=3)$.

MCF-7 tumor cells (24). Metformin has been reported to have angiogenic property (12), while its anti-cancer efficacy has also been well documented (25). Our data suggest that the effect of these five anti-PE drugs on the mini-tumor spheroids is attributed to their anti-cancer properties rather than their pro-angiogenic ones.

Contrary to these observations, nicorandil increased the size of the mini-tumor sprout treated with or without BEV. Nicorandil is an anti-angina drug with a dual mechanism of action, NO donor and ATP-sensitive potassium channel $\left(\mathrm{K}_{\mathrm{ATP}}\right)$ opener. To investigate the mechanisms underlying the action of nicorandil on the mini-tumor, diazoxide, a $\mathrm{K}_{\mathrm{ATP}}$ channel activator, was used to evaluate the invasion of mini- tumor spheroids, resulting in similar observations to when mini-tumors were treated with nicorandil (Figure 3). Besides, the increase in invasion of mini-tumors following treatment with nicorandil or diazoxide could be completely quenched by treatment with glibenclamide, a $\mathrm{K}_{\mathrm{ATP}}$ channel inhibitor. These data clearly indicate that $\mathrm{K}_{\text {ATP }}$ channel activation is responsible for the stimulatory effect of nicorandil on the mini-tumor spheroids.

Umaru et al. have recently shown that activation of $\mathrm{K}_{\mathrm{ATP}}$ channel induces angiogenesis in vitro and in vivo by performing experiments using endothelial cells and a chick chorioallantoic membrane model (17). In the case of cancer, $\mathrm{Ru}$ et al. have shown that $\mathrm{K}_{\mathrm{ATP}}$ inhibition exerts anti-tumor 
effects, while $\mathrm{K}_{\mathrm{ATP}}$ activation by diazoxide does not lead to tumor cell survival when cells are cultured in 2D conditions (26). Our results demonstrated that $\mathrm{K}_{\mathrm{ATP}}$ activation by nicorandil and diazoxide directly stimulated mini-tumor invasion in 3D culture, which is primarily due to the increased invasion of tumor cells, and not of endothelial cells or fibroblasts (Figure 4). Interestingly, when MDA-MB-231 cancer cells were independently treated with nicorandil or diazoxide on conventional 2D culture plates, there was no increase in proliferation (data not shown). These observations suggest that formation of a 3D structure and/or interaction with other cell types helps activating tumor cell behavior.

Our results provide new evidence that both nicorandil and diazoxide, the $\mathrm{K}_{\mathrm{ATP}}$ channel activators, induce invasion of mini-tumors in a $3 \mathrm{D}$ cultured model and may weaken the action of BEV. To our knowledge, there has been no previous report concerning the relationship between the clinical use of nicorandil and tumor progression or its effect on lowering the efficacy of BEV. Katoh et al. recently reported a case where nicorandil was effectively used for the management of microvascular angina pectoris caused by BEV for the treatment of ovarian cancer (27). This observation suggests that the pharmacological influence of these drugs could be partly counteracted by a yin and yang relationship.

In summary, we demonstrated that, by using a human minitumor co-cultured model, nicorandil was shown to enhance invasion of mini-tumors via $\mathrm{K}_{\mathrm{ATP}}$ channel activation and reduce the action of $\mathrm{BEV}$ in vitro. Although the current data are restricted to an artificial environment, they raised the possibility that clinical use of nicorandil may be associated with the outcome of cancer therapy. Further in vivo studies and the investigation of clinical data to validate the influence of the concomitant use of both drugs are needed.

\section{Conflicts of Interest}

The Authors declare that they have no competing interests.

\section{Authors' Contributions}

KO conceived the study and was in charge of overall direction and planning and critically revised the manuscript. CP designed and performed the experiments, analyzed data and was major contributor in writing the manuscript. TH gave advice on the experiments and writing of the manuscript and critically revised the manuscript. KO supervised the study. All Authors have read and approved the final manuscript.

\section{Acknowledgements}

The Authors would like to thank Shunji Harada for his technical assistance.

Funding: This work was supported by JSPS KAKENHI Grant Number JP19K07207.

\section{References}

1 Eskens FA and Sleijfer S: The use of bevacizumab in colorectal, lung, breast, renal and ovarian cancer: Where does it fit? Eur J Cancer 44: 2350-2356, 2008. PMID: 18789679. DOI: 10.1016/j.ejca.2008.07.042

$2 \mathrm{Li} \mathrm{M}$ and Kroetz DL: Bevacizumab-induced hypertension: Clinical presentation and molecular understanding. Pharmacol Ther 182: 152-160, 2018. PMID: 28882537. DOI: 10.1016/ j.pharmthera.2017.08.012

3 Jim B and Karumanchi SA: Preeclampsia: Pathogenesis, prevention, and long-term complications. Semin Nephrol 37: 386-397, 2017. PMID: 28711078. DOI: 10.1016/j.semnephrol. 2017.05.011

4 Karumanchi SA: Angiogenic factors in preeclampsia: From diagnosis to therapy. Hypertension 67: 1072-1079, 2016. PMID: 27067718. DOI: 10.1161/HYPERTENSIONAHA.116.06421

5 Thadhani R, Hagmann H, Schaarschmidt W, Roth B, Cingoez T, Karumanchi SA, Wenger J, Lucchesi KJ, Tamez H, Lindner T, Fridman A, Thome U, Kribs A, Danner M, Hamacher S, Mallmann P, Stepan H and Benzing T: Removal of soluble Fmslike tyrosine kinase-1 by Dextran sulfate apheresis in Preeclampsia. J Am Soc Nephrol 27: 903-913, 2016. PMID: 26405111. DOI: 10.1681/ASN.2015020157

6 McKeeman GC, Ardill JES, Caldwell CM, Hunter AJ and McClure N: Soluble vascular endothelial growth factor receptor1 (sFlt-1) is increased throughout gestation in patients who have preeclampsia develop. Am J Obstet Gynecol 191: 1240-1246, 2004. PMID: 15507947. DOI: 10.1016/j.ajog.2004.03.004

7 Kumasawa K, Ikawa M, Kidoya H, Hasuwa H, Saito-Fujita T, Morioka Y, Takakura N, Kimura T and Okabe M: Pravastatin induces placental growth factor (PGF) and ameliorates preeclampsia in a mouse model. Proc Natl Acad Sci 108: 14511455, 2011. PMID: 21187414. DOI: 10.1073/pnas.1011293108

8 Onda K, Tong S, Beard S, Binder N, Muto M, Senadheera SN, Parry L, Dilworth M, Renshall L, Brownfoot F, Hastie R, Tuohey L, Palmer K, Hirano T, Ikawa M, Kaitu'u-Lino T and Hannan NJ: Proton pump inhibitors decrease soluble fms-like tyrosine kinase- 1 and soluble endoglin secretion, decrease hypertension, and rescue endothelial dysfunction. Hypertension 69: 457-468, 2017. PMID: 28115513. DOI: 10.1161/ HYPERTENSIONAHA.116.08408

9 Burke SD, Zsengellér ZK, Khankin E V, Lo AS, Rajakumar A, DuPont JJ, McCurley A, Moss ME, Zhang D, Clark CD, Wang A, Seely EW, Kang PM, Stillman IE, Jaffe IZ and Karumanchi SA: Soluble fms-like tyrosine kinase 1 promotes angiotensin II sensitivity in preeclampsia. J Clin Invest 126: 2561-2574, 2016. PMID: 27270170. DOI: 10.1172/JCI83918

10 Li F, Fushima T, Oyanagi G, Townley-Tilson HWD, Sato E, Nakada H, Oe Y, Hagaman JR, Wilder J, Li M, Sekimoto A, Saigusa D, Sato H, Ito S, Jennette JC, Maeda N, Karumanchi SA, Smithies O and Takahashi N: Nicotinamide benefits both mothers and pups in two contrasting mouse models of preeclampsia. Proc Natl Acad Sci 113: 13450-13455, 2016. PMID: 27821757. DOI: 10.1073/pnas.1614947113

11 Syngelaki A, Nicolaides KH, Balani J, Hyer S, Akolekar R, Kotecha R, Pastides A and Shehata H: Metformin versus placebo in obese pregnant women without diabetes mellitus. N Engl J Med 374: 434-443, 2016. PMID: 26840133. DOI: 10.1056/ NEJMoa1509819 
12 Brownfoot FC, Hastie R, Hannan NJ, Cannon P, Tuohey L, Parry LJ, Senadheera S, Illanes SE, Kaitu'U-Lino TJ and Tong $S$ : Metformin as a prevention and treatment for preeclampsia: Effects on soluble fms-like tyrosine kinase 1 and soluble endoglin secretion and endothelial dysfunction. Am J Obstet Gynecol 214: 356.e1-356.e11, 2016. PMID: 26721779. DOI: 10.1016/j.ajog.2015.12.019

13 Stupakova EG, Lazareva GA and Gureev VV: Correction of morphofunctional disturbances arising when modelling preeclampsia with resveratrol and nicorandil. Res Results Pharmacol 4: 59-71, 2018. DOI: 10.3897/rrpharmacology. 4.25528

14 Cross SN, Ratner E, Rutherford TJ, Schwartz PE and Norwitz ER: Bevacizumab-mediated interference with VEGF signaling is sufficient to induce a preeclampsia-like syndrome in nonpregnant women. Rev Obstet Gynecol 5: 2-8, 2012. PMID: 22582121. DOI: $10.3909 / \operatorname{riog} 0179$

15 Correa de Sampaio P, Auslaender D, Krubasik D, Failla AV, Skepper JN, Murphy G and English WR: A heterogeneous in vitro three dimensional model of tumour-stroma interactions regulating sprouting angiogenesis. PLoS One 7: e30753, 2012. PMID: 22363483. DOI: 10.1371/journal.pone.0030753

16 Schneider CA, Rasband WS and Eliceiri KW: NIH image to ImageJ: 25 years of image analysis HHS public access. Nat Methods 9: 671675, 2012. PMID: 22930834. DOI: 10.1038/nmeth. 2089

17 Umaru B, Pyriochou A, Kotsikoris V, Papapetropoulos A and Topouzis S: ATP-sensitive potassium channel activation induces angiogenesis in vitro and in vivo. J Pharmacol Exp Ther 354: 7987, 2015. PMID: 25977483. DOI: 10.1124/jpet.114.222000

18 Amann A, Zwierzina M, Koeck S, Gamerith G, Pechriggl E, Huber JM, Lorenz E, Kelm JM, Hilbe W, Zwierzina H and Kern $\mathrm{J}$ : Development of a 3D angiogenesis model to study tumour endothelial cell interactions and the effects of anti-angiogenic drugs. Sci Rep 7: 1-13, 2017. PMID: 28592821. DOI: 10.1038/ s41598-017-03010-6

19 Yeh C, Cheng C-C, Lin H-C, Luo T-Y, Chang J and Ho A-S: Pravastatin inhibits tumor growth through elevating the levels of apolipoprotein A1. Adv Dig Med 3: 3-10, 2016. DOI: 10.1016/ j.aidm.2015.03.003

20 Menter DG, Ramsauer VP, Harirforoosh S, Chakraborty K, Yang P, Hsi L, Newman RA and Krishnan K: Differential effects of pravastatin and simvastatin on the growth of tumor cells from different organ sites. PLoS One 6, 2011. PMID: 22216116. DOI: 10.1371/journal.pone.0028813
21 Yeo M, Kim DK, Kim YB, Oh TY, Lee JE, Cho SW, Kim HC and Hahm KB: Selective induction of apoptosis with proton pump inhibitor in gastric cancer cells. Clin Cancer Res 10: 86878696, 2004. PMID: 15623654. DOI: 10.1158/1078-0432.CCR04-1065

22 Luciani F, Spada M, De Milito A, Molinari A, Rivoltini L, Montinaro A, Marra M, Lugini L, Logozzi M, Lozupone F, Federici C, Iessi E, Parmiani G, Arancia G, Belardelli F and Fais $\mathrm{S}$ : Effect of proton pump inhibitor pretreatment on resistance of solid tumors to cytotoxic drugs. J Natl Cancer Inst 96: 17021713, 2004. PMID: 15547183. DOI: 10.1093/jnci/djh305

23 Shi Z, Tiwari AK, Patel AS, Fu LW and Chen ZS: Roles of sildenafil in enhancing drug sensitivity in cancer. Cancer Res 71: 3735-3738, 2011. PMID: 21610107. DOI: 10.1158/00085472.CAN-11-0375

24 Wang T, Cui H, Ma N and Jiang Y: Nicotinamide-mediated inhibition of SIRT1 deacetylase is associated with the viability of cancer cells exposed to antitumor agents and apoptosis. Oncol Lett 6: 600-604, 2013. PMID: 24137378. DOI: 10.3892/ol.2013. 1400

25 Quinn BJ, Kitagawa H, Memmott RM, Gills JJ and Dennis PA: Repositioning metformin for cancer prevention and treatment. Trends Endocrinol Metab 24: 469-480, 2013. PMID: 23773243. DOI: $10.1016 /$ j.tem.2013.05.004

26 Li CY, Wu YX, Ru Q, Pi MS, Tian X and Wu RH: Voltage-gated and ATP-sensitive $\mathrm{K}+$ channels are associated with cell proliferation and tumorigenesis of human glioma. Oncol Rep 31: 842-848, 2013. PMID: 24284968. DOI: 10.3892/or.2013.2875

27 Katoh M, Abe H, Fujii T, Komuro I, Osuga Y, Takeda N, Arimoto $\mathrm{T}$ and Oda $\mathrm{K}$ : Bevacizumab-related microvascular angina and its management with nicorandil. Int Heart J 58: 803805, 2017. PMID: 28966326. DOI: 10.1536/ihj.16-537
Received April 26, 2019

Revised June 3, 2019

Accepted June 4, 2019 